\title{
Compensatory Activity in the Extrastriate Body Area of Parkinson's Disease Patients
}

\author{
Bart F. L. van Nuenen, ${ }^{1,2}$ Rick C. Helmich, ${ }^{1,2}$ Noud Buenen, ${ }^{1}$ Bart P. C. van de Warrenburg, ${ }^{1}$ Bastiaan R. Bloem, \\ and Ivan Toni ${ }^{2}$ \\ ${ }^{1}$ Radboud University Nijmegen Medical Center, Donders Institute for Brain, Cognition and Behaviour, Department of Neurology, 6500 HB Nijmegen, The \\ Netherlands, and ${ }^{2}$ Radboud University Nijmegen, Donders Institute for Brain, Cognition and Behaviour, Centre for Cognitive Neuroimaging, 6525 HR \\ Nijmegen, The Netherlands
}

Compensatory mechanisms are a crucial component of the cerebral changes triggered by neurodegenerative disorders. Identifying such compensatory mechanisms requires at least two complementary approaches: localizing candidate areas using functional imaging, and showing that interference with these areas has behavioral consequences. Building on recent imaging evidence, we use this approach to test whether a visual region in the human occipito-temporal cortex - the extrastriate body area-compensates for altered dorsal premotor activity in Parkinson's disease (PD) during motor-related processes. We separately inhibited the extrastriate body area and dorsal premotor cortex in 11 PD patients and 12 healthy subjects, using continuous theta burst stimulation. Our goal was to test whether these areas are involved in motor compensatory processes. We used motor imagery to isolate a fundamental element of motor planning, namely subjects' ability to incorporate the current state of their body into a motor plan (mental hand rotation). We quantified this ability through a posture congruency effect (i.e., the improvement in subjects' performance when their current body posture is congruent to the imagined movement). Following inhibition of the right extrastriate body area, the posture congruency effect was lost in PD patients, but not in healthy subjects. In contrast, inhibition of the left dorsal premotor cortex reduced the posture congruency effect in healthy subjects, but not in PD patients. These findings suggest that the right extrastriate body area plays a compensatory role in PD by supporting a function that is no longer performed by the dorsal premotor cortex.

\section{Introduction}

Neurodegenerative disorders are often associated with systemlevel compensatory phenomena, with behavioral impairments emerging from a cerebral balancing act between compensatory and degenerative processes: neurodegeneration starts several years before affected individuals start to display clinically visible symptoms, allowing for cerebral compensation to develop in unaffected brain areas (Braak et al., 2003; Palop et al., 2006). Characterizing cerebral compensatory phenomena is crucial for their potential therapeutic exploitation, and for understanding how different cerebral circuits can support the same function or yield the same output (Edelman and Gally, 2001).

Parkinson's disease (PD), a prototypical example of a neurodegenerative disorder, is characterized by degeneration of dopaminergic neurons in the substantia nigra pars compacta. This

Received Jan. 23, 2012; revised May 10, 2012; accepted May 22, 2012.

Author contributions: B.F.L.v.N., R.C.H., B.P.C.V.d.W., B.R.B., and I.T. designed research; B.F.L.v.N. and N.B. performed research; B.F.L.v.N. contributed unpublished reagents/analytic tools; B.F.L.v.N., R.C.H., N.B., B.R.B., and I.T. analyzed data; B.F.L.v.N., R.C.H., B.P.C.v.d.W., B.R.B., and I.T. wrote the paper.

This work was supported by The Netherlands Organisation for Scientific Research (NWO) VIDI Research Grant 016.076.352 (B.F.L.v.N., B.R.B.), NWO Brain and Cognition Grant 433.09.248 (I.T.), and the BrainGain Smart Mix Programme of The Netherlands Ministry of Economic Affairs/Ministry of Education, Culture and Science (I.T.).

The authors declare no competing financial interests.

Correspondence should be addressed to Prof. Bastiaan R. Bloem, Radboud University Nijmegen Medical Centre, Department of Neurology (935), P.0. Box 9 101, 6500 HB Nijmegen, The Netherlands. E-mail: b.bloem@ neuro.umen.nl.

DOI:10.1523/JNEUROSCI.0335-12.2012

Copyright $\odot 2012$ the authors $\quad 0270-6474 / 12 / 329546-08 \$ 15.00 / 0$ degeneration causes dopamine depletion and disrupts the function of both the basal ganglia and the connected neural circuits (e.g., the basal ganglia-thalamo-motor cortex circuit) (Blandini et al., 2000). Disruption in this circuit eventually leads to motor deficits, clinically apparent as the classic symptoms of PD: akinesia, hypokinesia, and bradykinesia (Berardelli et al., 2001). Observations in animal models of PD and in clinically unaffected humans at risk of developing PD (i.e., asymptomatic Parkin and PINK1 mutation carriers) have shown that, before the disease becomes symptomatic, compensatory mechanisms arise within the fronto-striatal circuit (Bezard et al., 2003; Buhmann et al., 2005; van Nuenen et al., 2009). After clinical signs have become evident, compensatory mechanisms appear to engage circuits involving more posterior sensory regions (Sabatini et al., 2000; Helmich et al., 2007). These changes might explain why PD motor deficits improve when external sensory cues are provided (Suteerawattananon et al., 2004; Azulay et al., 2006; Keus et al., 2009). Accordingly, we have recently shown that the right extrastriate body area (EBA), a visual region in the occipito-temporal cortex, showed stronger activity and connectivity with the left dorsal premotor cortex (PMd) when PD patients imagined moving their most-affected hand (Helmich et al., 2007). However, it remains difficult to prove that stronger EBA connectivity (as identified using functional imaging) is compensatory, rather than a collateral by-product of basal ganglia disinhibition. Further supportive evidence requires at least a second complemen- 
Table 1. Clinical characteristics

\begin{tabular}{lllllc}
\hline Patient & Gender & Age (years) & H\&Y & UPDRS-L & UPDRS-R \\
\hline 1 & W & 54 & 1.5 & 1 & 12 \\
2 & M & 46 & 1 & 0 & 3 \\
3 & M & 69 & 2 & 1 & 7 \\
4 & W & 50 & 1.5 & 1 & 7 \\
5 & M & 41 & 1 & 0 & 5 \\
6 & M & 46 & 1 & 3 & 10 \\
7 & M & 65 & 1 & 1 & 6 \\
8 & W & 52 & 1 & 0 & 7 \\
9 & W & 52 & 2 & 1 & 9 \\
10 & M & 54 & 1 & 0 & 5 \\
11 & W & 53 & 2 & 4 & 13 \\
Mean & 6 men & 52 & 1.4 & 1.1 & 7.6 \\
SD & & 7.8 & 0.5 & 1.3 & 3.1 \\
\hline
\end{tabular}

Eleven patients (six men; age, $52.0 \pm 7.8$ years; mean \pm SD) with idiopathic Parkinson's disease were tested in a practically defined off-state (i.e., $>12 \mathrm{~h}$ after having taken their last medication). All patients were consistent right-handers. Patients had markedly asymmetric symptoms lateralized to the right side of their body. UPDRS, Unified Parkinson's Disease Rating Scale; H\&Y, Hoehn and Yahr rating scale; M, man; W, woman.

tary approach: showing that interference with the candidate area identified by functional imaging has behavioral consequences.

We have used this approach to test the possibility that the EBA compensates for altered PMd activity in PD during motor-related processes. Specifically, we have used continuous theta burst stimulation (cTBS) (Huang et al., 2005) to selectively inhibit either EBA or PMd to test their possible compensatory role in PD. We assessed the consequences of this interference on both corticospinal excitability and cerebral motor function. The latter was indexed through a validated motor imagery task that quantifies subjects' ability to incorporate the current state of their body into a motor plan (de Lange et al., 2006; Helmich et al., 2007). This ability should be reduced if EBA or PMd play a compensatory role in PD.

\section{Materials and Methods}

Subjects. Eleven PD patients ( 6 men; $52.0 \pm 7.8$ years; mean \pm SD; Table 1 for clinical characteristics) and 12 healthy subjects ( 6 men; $61.3 \pm 6.4$ years; mean $\left.\pm \mathrm{SD} ; t_{(21)}=2.912 ; p=0.01\right)$ participated after giving informed consent according to institutional guidelines of the local ethics committee (CMO Region Arnhem-Nijmegen, The Netherlands). Participants were consistently right-handed according to the Edinburgh Handedness Inventory (Oldfield, 1971). Before the experiment, the patients' disease severity was assessed by one examiner (B. F. L. van Nuenen) using the Hoehn and Yahr stages and the Unified Parkinson's Disease Rating Scale (UPDRS) (Table 1). Patients were included if they had idiopathic PD, diagnosed according to the United Kingdom Brain Bank criteria by an experienced movement disorder specialist (B. R. Bloem), with clearly right-lateralized PD symptoms. Exclusion criteria were as follows: cognitive dysfunction (i.e., Mini Mental State Examination, <24), other neurological diseases (such as severe head trauma or stroke) and general exclusion criteria for transcranial magnetic stimulation (TMS) (such as epilepsy, pacemaker, implanted metal parts, and cardiac arrhythmias). Patients were all studied twice with at least a 1 week interval and off medication with at least a $12 \mathrm{~h}$ withdrawal of medication [i.e., practically defined off-condition (Langston et al., 1992)]. The whole experimental protocol involved two experimental sessions, with session order counterbalanced between participants. The experimental sessions were identical apart from the cTBS protocol, which used either cTBS over the right EBA or the left PMd. At least $7 \mathrm{~d}$ elapsed between the two sessions to exclude carry-over effects of TBS conditioning. Figure $1 A$ illustrates the order of the experimental procedures. At the beginning of each experimental session, participants were trained on the motor imagery task (192 trials and $<10 \%$ of errors; see below for task description). Afterward, cortical excitability of the left primary motor hand area (M1) was probed with single-pulse TMS of left M1, recording motor evoked potentials (MEPs) from the right first dorsal interosseus (FDI) muscle. Following performance of a set of 384 motor imagery trials, cortical excitability in left M1 was measured again. Following application of cTBS to either the left PMd or the right EBA (40 s), participants rested for 7 min without moving their hands or feet. We introduced this resting period because previous studies have shown that short periods of voluntary motor activity shortly before or after cTBS can strongly modulate the conditioning effects of cTBS on cortical excitability (Huang et al., 2005; Gentner et al., 2008). Following this resting period, cortical excitability in left M1 was measured again to capture acute TBS-induced changes in corticospinal excitability. Thereafter, participants performed the motor imagery task again (384 trials). The experimental session was completed with a fourth measurement of cortical excitability with single-pulse TMS over the left M1.

Motor imagery task. We asked participants to perform a hand laterality judgment task (i.e., a task that has been repeatedly shown to reliably evoke motor imagery) (de Lange et al., 2006; Helmich et al., 2007). Participants were shown line drawings of one hand at a time, either left or right, with either the back or the palm of the hand in view. The left and right hand drawings were identical mirror images. A hand drawing could be shown rotated from its upright position in either a counterclockwise (CCW) or a clockwise (CW) orientation. For both orientations, four different rotations from 45 to $135^{\circ}$ in steps of $30^{\circ}$ were used, yielding eight different rotations. The stimuli were presented through a PC running Presentation software (Neurobehavioral Systems). They were projected onto a screen in front of the subjects. The subjects' task was to report whether the hand drawing on display represented a left or a right hand by pressing one of the two buttons with the corresponding left or right foot. During the task, reaction times (RTs) and error rates were measured. One experimental session consisted of 32 blocks. Each block consisted of 12 trials, which started with a fixation cross displayed for a variable interval $(0.5-1.5 \mathrm{~s})$, followed by the presentation of a hand drawing. After a response was provided (reaction time cutoff, $5 \mathrm{~s}$ ), the stimulus was replaced by the fixation cross for a jittered period of $0.5-1.5 \mathrm{~s}$, and then the next hand drawing was shown. On each trial, a different hand drawing was presented, pseudorandomly sampled from a set of 32 . During the experiment, the posture of the subjects' left and right hand was manipulated. At the beginning of each block, a text instructed the subjects to position their arms in one of four postures: (1) both hands with the palm up; (2) left hand palm up, right hand palm down; (3) left hand palm down, right hand palm up; (4) both hands palm down. The period during which this instruction was displayed had a fixed duration ( $5 \mathrm{~s})$ and was followed by a block of 12 trials. The posture effect was established by comparing the presented hand drawing and the actually instructed posture of the subject. The posture for a trial was coded as "matching" when the side of the hand (palm or back) and the laterality (left or right) of the hand drawing corresponded with the hand position of the subject. When the hand drawing and the hand position did not correspond, the posture for the trial was coded as "nonmatching." We also considered the influence of biomechanical constraints (BMCs) on motor imagery performance. BMCs were operationalized as the RT difference in mentally rotating a hand toward either an orientation lateral to the body axis (i.e., toward the extreme range of movement allowed by the arm joints) or toward an orientation medial to the body axis (i.e., toward a comfortable arm configuration). Lateral and medial orientations were coded as follows: CCW rotations $\left(-135,-105,-75\right.$, and $\left.-45^{\circ}\right)$ were averaged and recoded as a lateral orientation for left hands and a medial orientation for right hands; CW rotations $\left(45,75,105,135^{\circ}\right)$ were averaged and recoded as a medial orientation for left hands and a lateral orientation for right hands. Participants were seated in front of the screen with their hands in a box $(75 \times 27 \times 9.5 \mathrm{~cm})$, so there was no visual feedback of their own hands. Before the start of the experiment, patients were trained until they could perform the task with an accuracy of at least $90 \%$ correct responses.

Continuous theta burst stimulation of left PMd and right EBA. We used cTBS for conditioning of left PMd and right EBA because cTBS has been shown to produce a lasting suppression of regional excitability in the stimulated cortex (Huang et al., 2005). The cTBS protocol involved repeated administration of short high-frequency bursts. Each burst consisted of three pulses given at an interstimulus interval of $20 \mathrm{~ms}$ 
(corresponding to a rate of $50 \mathrm{~Hz}$ ). These highfrequency triple-pulse bursts were repeated every $200 \mathrm{~ms}$. Theta burst stimulation was given to left PMd as a continuous train lasting for $40 \mathrm{~s}$. The intensity of cTBS was set at $80 \%$ of the individual active motor threshold. In every subject, cTBS was delivered over the right EBA and over the left PMd in two different sessions with a minimal interval of $7 \mathrm{~d}$. The location in the first session was randomized, so in the first session one-half of the subjects and one-half of the patients had cTBS over the right EBA and the other subjects had cTBS over the left PMd. The site for left PMd stimulation was $2 \mathrm{~cm}$ anterior and $1 \mathrm{~cm}$ medial to the "motor hot spot" on the left motor cortex. The site for right EBA stimulation was determined on the basis of the following procedure. We performed a pilot in five subjects (four men), in which the stereotactic coordinates of the right EBA, as obtained in Helmich et al. (2007) (MNI coordinates: [ $x y$ $z]=[+46-78+6])$ were mapped onto $\mathrm{T} 1$ structural scans of those subjects. Each individual right EBA location was then projected on the skull with a stereotactic image guidance system (Brainsight; Rogue Research). This procedure revealed that the skull projection of the right EBA had a consistent location across the five subjects, namely $12 \mathrm{~cm}$ laterally and $7 \mathrm{~cm}$ posterior to the vertex $(\mathrm{Cz})$. Accordingly, we used this skull-based coordinates to localize EBA in the participants tested in this study. For both simulation sites, the coil was positioned tangentially to the skull with the handle pointing backward and laterally at an angle of $\sim 45^{\circ}$ to the sagittal plane (Urgesi et al., 2004, 2007).

Analysis of task performance. We considered two outcome measures of the effects of cTBS on PMd and EBA: one behavioral and one physiological outcome measure. The behavioral outcome was performance of the motor imagery task, and more specifically how motor features like biomechanical constraints and the current hand posture influenced reaction times. Behavioral data were analyzed using SPSS Statistics 19.0. Incorrect responses (either wrong or no response) were excluded from further analysis. We considered RTs of the correct responses (measured in milliseconds) and number of errors (Errors [i.e., errors/total number of trials (\%)]). Errors and RT from both EBA and PMd preintervention sessions within each group were combined as a single baseline (paired-samples $t$ test on Errors and RT on these sessions did not reveal any significant difference). First, we tested whether participants from the two groups performed the motor imagery task appropriately (before receiving cTBS), and whether they did so in a comparable manner. We analyzed the influence of the factors Group (two levels: Control or Patient), Laterality (two levels: Right or Left), Orientation (two levels: Lateral or Medial), Posture (two levels: Match or Nonmatch), and Rotation (eight levels: from $-135^{\circ}$ to $-45^{\circ}$ and $45^{\circ}$ to $135^{\circ}$ in steps of $30^{\circ}$ ), by means of a five-way repeated-measures ANOVA on RT collected during the baseline sessions. Second, we analyzed how cTBS over EBA or PMd influenced overall reaction times and error rates, by assessing the effects of factors Group (two levels: Control or Patient), Session (three levels: baseline, post-EBA session, post-PMd session) by means of two two-way repeated-measures ANOVAs on errors and RTs. Third, we tested separately the effect of BMCs and hand posture (HP) on behavioral performance. Specifically, we tested the effect of factors Group (two levels: healthy subjects or patients), Session (three levels: baseline, post-EBA session, or post-PMd session), and Posture (match or nonmatch) or Orientation (lateral or medial) on the RT using two three-way repeated-measures ANOVAs. The GreenhouseGeisser method was used to correct for nonsphericity. The $\alpha$ level was set at $p=0.05$.
Measurement and analysis of corticospinal excitability. The physiological outcome measure of cTBS effects was corticospinal excitability, assessed with single-pulse TMS over the left M1 using a biphasic pulse configuration and a 70-mm-diameter figure-of-eight shaped coil (Magstim Company Ltd.) connected to a Magstim Super Rapid stimulator (Magstim Company Ltd.). TMS was applied while participants were comfortably seated in an armchair. Both arms were supported by a cushion to facilitate complete relaxation of the arm and hand muscles. Subjects were instructed to relax but to keep their eyes open and fixate on a wall $1.5 \mathrm{~m}$ in front of them.

The coil was positioned tangentially to the skull over the left $\mathrm{M} 1_{\mathrm{Hand}}$ with the handle pointing backward and laterally at an angle of $\sim 45^{\circ}$ to the sagittal plane. At this coil orientation, the second phase of the biphasic TMS pulse induces an electrical current in the brain tissue with a posterior-lateral to anterior-medial direction roughly perpendicular to the central sulcus, which is optimal for evoking a motor response in the contralateral hand (Mills et al., 1992).

We defined the scalp site where a single TMS pulse at slightly suprathreshold intensity consistently yielded maximal MEP in the right contralateral FDI muscle. This "motor hot spot" was used as stimulation site for all TMS measurements and used as anchor point to define the site for TBS of the left PMd. To individually adjust the stimulus intensity, we determined the resting and active motor thresholds (MTs). We first determined the resting MT in the relaxed FDI muscle, which was defined as the minimum stimulus intensity that produced an MEP of $>50 \mu \mathrm{V}$ in 5 of 10 consecutive trials. We then measured the active MT defined as the lowest stimulus intensity at which MEPs were elicited in 5 of 10 consecutive trials during tonic contraction of the FDI muscle at $\sim 10 \%$ of maximum force level using a criterion for the MEP of 100 $250 \mu \mathrm{V}$ peak-to-peak amplitude. MTs were determined by gradually decreasing and increasing the stimulus intensity in steps of $1 \%$ of maximum stimulator output. 


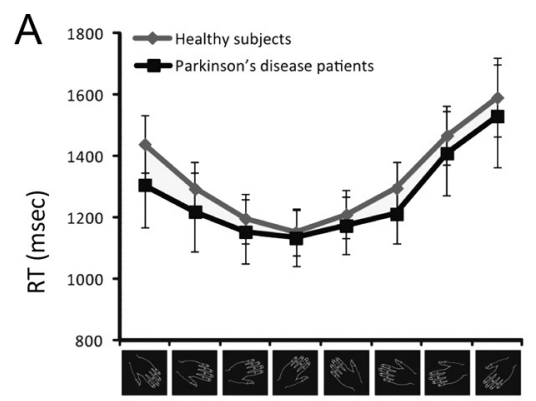

B
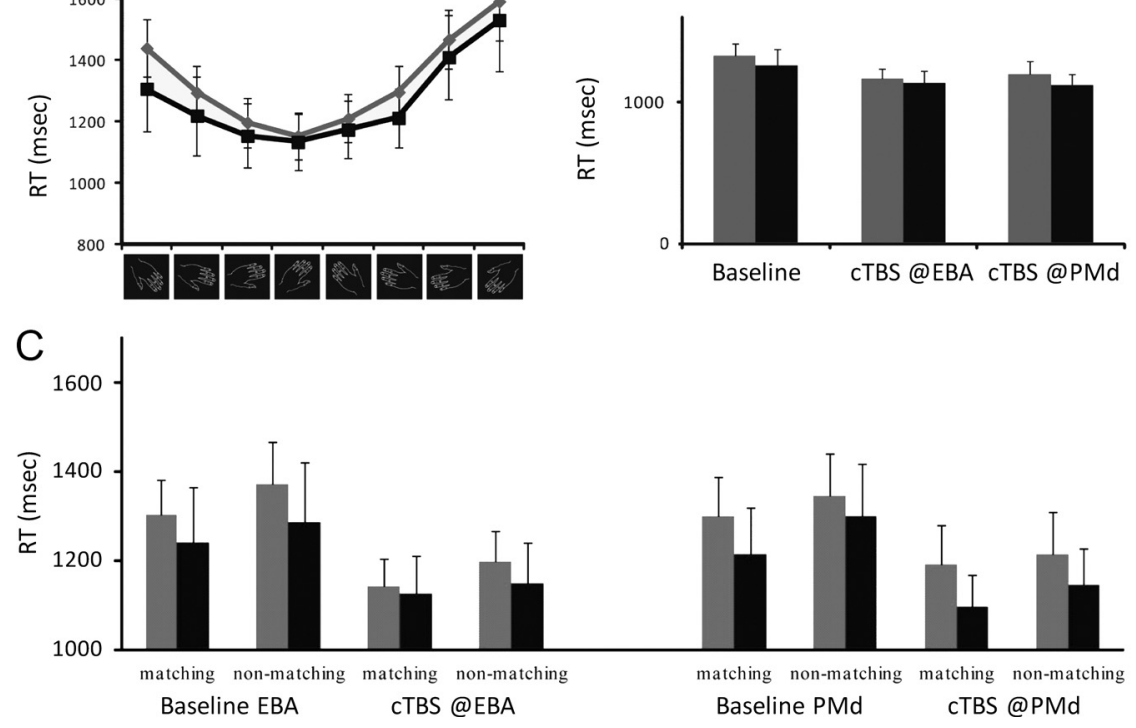

Figure 2. Behavioral performance. $A$, Response times (mean \pm SEM) during the baseline sessions as a function of group (healthy subjects or Parkinson's disease patients) and stimulus rotation (as illustrated by the hand drawings). Response times changed as a function of stimulus rotation for both groups. $\boldsymbol{B}$, Response times (mean $\pm \mathrm{SEM}$ ) as a function of group (healthy subjects or Parkinson patients) and experimental session (baseline, after cTBS over EBA, and after CTBS over PMd). Response times decreased after either CTBS interventions, similarly for both groups. C, Response times (mean \pm SEM) as a function of hand posture (healthy subjects or Parkinson patients) and experimental session (baseline, after CTBS over EBA, and after (TBS over PMd).

MEPs were recorded with surface electromyography (EMG). $\mathrm{Ag}-\mathrm{AgCl}$ disc surface electrodes were attached over the right FDI muscle using a bellytendon montage. The grounding electrode was placed at the wrist. Electromyographic activity was continuously monitored using visual (oscilloscope) and auditory (speakers) EMG feedback to ensure either complete relaxation at rest or a constant level of EMG activity during tonic contraction. The raw EMG signals were filtered between 20 and $1000 \mathrm{~Hz}$, and digitized at $5000 \mathrm{~Hz}$ per channel (A/D converter; model Micro1401; Cambridge Electronic Design). The administration of TMS pulses as well as EMG data recording, storage, and analyses were performed with Spike2 software (Cambridge Electronic Design). To measure corticospinal excitability, we applied 20 pulses with a mean of $0.2 \mathrm{~Hz}$ (with random interstimulus intervals of 4, 5 , or $6 \mathrm{~s}$ ) and an intensity necessary to obtain a $1 \mathrm{mV}$ MEP in the contralateral FDI. After baseline recording, this was repeated four times, before and after each motor imagery task (Fig. $1 B$ ).

Peak-to-peak amplitudes (in millivolts) of the MEP recorded from the right FDI muscle were measured trial-by-trial and mean MEP amplitudes were calculated for each block of measurements (MATLAB software; Mathworks). First, we conducted a paired-samples $t$ test for the MEPs for each group between the two preintervention sessions. When there were no significant differences between the two preintervention sessions, we further combined them as one baseline mean for each group and each intervention. Repeated-measures ANOVA was used to test for lasting effects of cTBS over left PMd or right EBA on excitability of ipsilateral left $\mathrm{M}_{\mathrm{Hand}}$. The ANOVA model included the factors Group (two levels: healthy subject or PD), Intervention (two levels: $\mathrm{cTBS}_{\mathrm{EBA}}$ or $\mathrm{CTBS}_{\mathrm{PMd}}$ ), and Block of Measurement [three levels: baseline, measurements starting $7 \mathrm{~min}$ after cTBS (cTBS1) or after last MI session (cTBS2)]. ANOVA was followed by post hoc two-sided paired-sample $t$ tests conditional of significant $F$ values. For all analyses, a significance level of $p<0.05$ was applied after nonsphericity (Greenhouse-Geisser) correction.

\section{Results}

Patients

The patients had markedly lateralized symptoms according the UPDRS (two-samples $t$ test: $t_{(10)}=9.8 ; p<0.001$; Table 1 ) and were on average 9.3 years younger than the healthy subjects $\left(t_{(21)}=2.9\right.$; $p=0.01)$.

\section{Behavioral performance at baseline Overall performance}

Patients and healthy subjects performed the task accurately, with mean error rates and mean reaction times over all sessions that were comparable across groups (mean error rates \pm SEM: healthy subjects, $2.3 \pm 0.7 \%$; patients, $3.3 \pm 1.1 \%$; two-samples $t$ test: $t_{(21)}=-0.407 ; p=$ 0.688 ; mean RT \pm SEM: healthy subjects, $1257 \pm 81 \mathrm{~ms}$; patients, $1194 \pm 97 \mathrm{~ms}$; two-samples $t$ test: $t=0.505 ; p=0.619$ ).

\section{Mental rotation performance}

RTs changed as a function of stimulus rotation (main effect of Rotation: $F_{(7,19)}=$ 32.38; $p<0.001$ ), and this effect was comparable across groups (interaction Group by Rotation: $F_{(7,2)}=0.40 ; p=0.636$; Fig. $2 A)$. RTs increased also as a function of Laterality $\left(F_{(1,11)}=18.82 ; p=0.001\right)$, Orientation $\left(F_{(1,11)}=42.71 ; p<0.001\right)$, and Posture $\left(F_{(1,11)}=20.85 ; p=0.001\right)$. There were no significant interactions of Group with other factors $(p>0.1)$. These findings are in line with previous studies, and they imply that both patients and healthy subjects were engaged in motor imagery, taking the current position of their hand and the biomechanical constraints of their joints into account when solving the hand laterality judgment task (de Lange et al., 2006; Helmich et al., 2007).

\section{Behavioral changes induced by cTBS \\ Overall performance}

There were no significant between-groups differences in error rates and RT before the CTBS intervention (baseline session). The mean error rate did not significantly change between sessions (main effect of Session: $F_{(2,18)}=4.29 ; p=0.32$ ), and there were no significant between-sessions differences across groups (Session by Group interaction: $F_{(2,15)}=0.013 ; p=$ 0.970 ). The mean RT changed between sessions (main effect of Session: $\left.F_{(2,19)}=18.39 ; p<0.001\right)$, indicating a time-related effect that, importantly, did not differ between groups (Group by Session interaction: $F_{(2,19)}=0.43 ; p=0.653$; Fig. $\left.2 B\right)$.

\section{Mental rotation performance}

To assess whether cTBS altered movement-related processes, we considered how BMCs and HP influenced cTBS effects on task performance. Given the presence of time-related effects across sessions (see previous paragraph), we focused these analyses on differential RT effects sampled within the same experimental session. For each session, we considered the RT differences between lateral and medial hand orientations (BMC $\triangle \mathrm{RT}$ ) and the difference in RT between matching and nonmatching hand postures (HP $\triangle \mathrm{RT}$ ). We found that cTBS over either EBA or PMd had opposite effects on the ability of patients or healthy subjects to incorporate their hand posture into imagined movements (Group by Session interaction on HP $\Delta \mathrm{RT}: F_{(2,18)}=7.52 ; p=$ 0.005 ; Fig. 3). Paired-samples $t$ test revealed that, in the PD group, EBA-cTBS significantly reduced the effect of hand posture on 


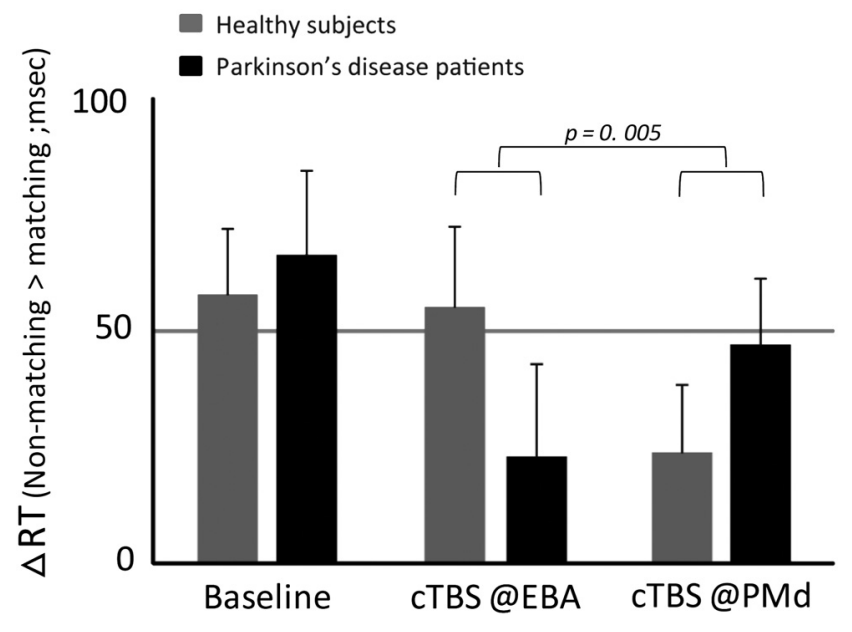

Figure 3. Behavioral performance: effects of cTBS. Differences in RTs (mean in milliseconds \pm SEM) between trials with nonmatching and matching configurations between subjects' own hands and hand drawings on display (posture congruency effect). Data are shown as a function of group and experimental session. CTBS over PMd reduced the posture congruency effect in the healthy subject group. cTBS over EBA reduced the posture congruency effect in the PD group.

imagery performance in the PD group compared with baseline (HP $\Delta$ RT: $t_{(10)}=2.55 ; p=0.029$ ). In the control group, PMdcTBS significantly reduced the effect of hand posture on imagery performance compared with EBA-cTBS (HP $\Delta \mathrm{RT}: t_{(11)}=2.29$; $p=0.043$; Fig. 3 ). This interaction was not driven by a speedaccuracy trade-off in the posture effects (Group by Session by Posture interaction on error rate: $\left.F_{(2,20)}=0.997 ; p=0.385\right)$.

There were no significant between-group differential effects of cTBS intervention when considering biomechanical constraints (Group by Session interaction on BMC $\Delta$ RT: $F_{(2,12)}=0.056 ; p=$ $0.878)$. These findings were confirmed in additional analyses performed on the absolute RTs (values shown in Table 2), showing that cTBS over either EBA or PMd had opposite effects on the ability of patients or healthy subjects to incorporate their hand posture into imagined movements (Group by Session by Posture interaction on RT: $\left.F_{(2,20)}=17.78 ; p<0.001\right)$. Paired-samples $t$ test revealed that, in the PD group, EBA-cTBS diminished the effect of hand posture on imagery performance in the PD group [HP (match vs nonmatch): $\left.t_{(10)}=-1.16 ; p=0.272\right]$ compared with PMd-cTBS [HP (match vs nonmatch): $t_{(10)}=-3.27 ; p=$ 0.008 ] and baseline [HP (match vs nonmatch): $t_{(10)}=-3.60 ; p=$ 0.005]. In the control group, PMd-cTBS diminished the effect of hand posture on imagery performance [HP (match vs nonmatch): $\left.t_{(11)}=-1.65 ; p=0.127\right]$ compared with EBA-cTBS [HP (match vs nonmatch): $\left.t_{(11)}=-3.13 ; p=0.010\right]$ and baseline [HP (match vs nonmatch): $\left.t_{(11)}=-3.97 ; p=0.002\right]$. Again, there were no significant between-group differential effects of cTBS intervention when considering biomechanical constraints (Group by Session by Orientation interaction on RT: $F_{(2,20)}=$ $0.056 ; p=0.878)$.

Finally, there were no significant between-sessions effects (Session on BMC $\Delta$ RT: $F_{(2,15)}=3.06 ; p=0.087$; Session on HP $\Delta$ RT: $F_{(2,20)}=2.53 ; p=0.107$ ) or laterality effects (Hand on BMC $\Delta$ RT: $F_{(1,10)}=1.06 ; p=0.328$; Hand on HP $\Delta$ RT: $F_{(1,10)}=$ $0.12 ; p=0.773)$. There were no significant interactions of Hand with other factors ( $p>0.1$ for all interactions).

It is possible that the altered task performance observed after cTBS over EBA in the PD group could be driven by an impairment of recognizing whether the hand picture shows a palm or a back view, rather than a visuomotor impairment. We tested this possibility with a three-way repeated-measurement ANOVA with the factors Group (healthy subjects or patients), Session (baseline or EBA or PMd), and hand orientation (Back or Palm) on RTs. These three factors did not significantly interact $\left(F_{(2,220)}=0.74 ; p=0.463\right)$, indicating that cTBS over EBA does not differentially impair the recognition of palm or back views of hands in the two groups of subjects.

\section{MEPs}

The two baseline MEP measurements before cTBS were not significantly different within each of the two groups. This indicates that performing the task did not influence corticospinal excitability of the left M1. In both groups (PD and healthy subjects), and for both sites of stimulation (EBA and PMd), cTBS led to an initial reduction of corticospinal excitability that was followed by increased corticospinal excitability after motor imagery performance $\left(F_{(2,17)}=5.0 ; p=0.023\right)$. Post hoc paired-samples $t$ test revealed a significant decrease in MEPs for the PD group in the first post-PMd session compared with the baseline measurements, but not for the healthy subjects (healthy subjects: $p=$ 0.195 ; PD patients: $p=0.027$; Fig. 4 ) and an increase in MEPs for the healthy control group after the motor imagery task after cTBS over PMd compared with the baseline measurements, but not for the PD patients (healthy subjects: $p=0.028$; PD patients: $p=$ 0.119; Fig. 4). There were no significant differences after cTBS over EBA for both groups. Finally, given the variability observed in the baseline measurements (Fig. 4), we considered whether the MEP effects we report are present also against session-specific baselines. We performed an additional ANOVA with the two baseline measurements separated, considering the factors Group (PD or healthy subjects), Intervention (PMd cTBS or EBA cTBS), and Time (pre-cTBS or post-cTBS). This ANOVA revealed an Intervention by Time interaction $\left(F_{(1,9)}=5.8 ; p=0.036\right)$ and a Time effect $\left(F_{(1,9)}=6.2 ; p=0.035\right)$. Post hoc paired $t$ tests revealed no significant differences between baseline MEP data $(p>$ 0.270 ). There were no significant differences with the two baseline measurements.

\section{Discussion}

We assessed a possible compensatory role of the EBA during motor imagery in PD patients, as suggested by a previous fMRI study (Helmich et al., 2007), by testing whether inhibition of this area (using cTBS) influences behavioral performance in PD. This was done by using a validated task that quantifies subjects' ability to consider their current body posture when imagining a movement (de Lange et al., 2006; Helmich et al., 2007). There are two main results. First, after inhibition of the right EBA, PD patients were unable to benefit from knowledge of their hand posture during motor imagery, as indicated by absence of a posture congruency effect (de Lange et al., 2006). The same intervention had no effect in healthy subjects. This suggests that, unlike healthy subjects, PD patients depend upon EBA for providing the motor system with an estimate of the current state of the body in space (derived from somatosensory information, given that subjects could not see their hands during the experiment). Healthy subjects did not require this compensatory activity from EBA or, alternatively, engaged other brain regions to compensate for the transient EBA alteration. Second, inhibition of the left PMd reduced the posture congruency effect in healthy subjects, but not in $\mathrm{PD}$ patients. We infer that, in $\mathrm{PD}$, the $\mathrm{PMd}$ is functionally disconnected from the cerebral network incorporating the current state of the body in space into a motor plan. The right EBA apparently compensates for this PMd alteration, and this was 
Table 2. Reaction times

\begin{tabular}{|c|c|c|c|c|c|c|}
\hline & \multicolumn{2}{|l|}{ Baseline } & \multicolumn{2}{|l|}{$\mathrm{EBA}$} & \multicolumn{2}{|l|}{ PMd } \\
\hline & Healthy subjects & PD & Healthy subjects & PD & Healthy subjects & $P D$ \\
\hline RT overall & $1.33(0.30)$ & $1.26(0.38)$ & $1.17(0.23)$ & $1.15(0.30)$ & $1.20(0.32)$ & $1.12(0.26)$ \\
\hline \multicolumn{7}{|l|}{ Laterality } \\
\hline Right & $1.27(0.27)$ & $1.25(0.36)$ & $1.11(0.21)$ & $1.12(0.29)$ & $1.16(0.30)$ & $1.09(0.26)$ \\
\hline Left & $1.39(0.32)$ & $1.27(0.38)$ & $1.24(0.25)$ & $1.15(0.31)$ & $1.25(0.33)$ & $1.15(0.25)$ \\
\hline \multicolumn{7}{|l|}{ Posture } \\
\hline Match & $1.30(0.28)$ & $1.23(0.36)$ & $1.12(0.21)$ & $1.07(0.30)$ & $1.16(0.32)$ & $1.07(0.27)$ \\
\hline Nonmatch & $1.36(0.31)$ & $1.29(0.40)$ & $1.22(0.26)$ & $1.20(0.30)$ & $1.24(0.31)$ & $1.17(0.26)$ \\
\hline \multicolumn{7}{|l|}{ Orientation } \\
\hline Lateral & $1.39(0.32)$ & $1.32(0.39)$ & $1.22(0.26)$ & $1.20(0.31)$ & $1.24(0.31)$ & $1.17(0.26)$ \\
\hline Medial & $1.27(0.28)$ & $1.20(0.37)$ & $1.12(0.21)$ & $1.07(0.30)$ & $1.16(0.32)$ & $1.07(0.27)$ \\
\hline
\end{tabular}

Shown are reaction times [mean (in seconds) and SD in parentheses].

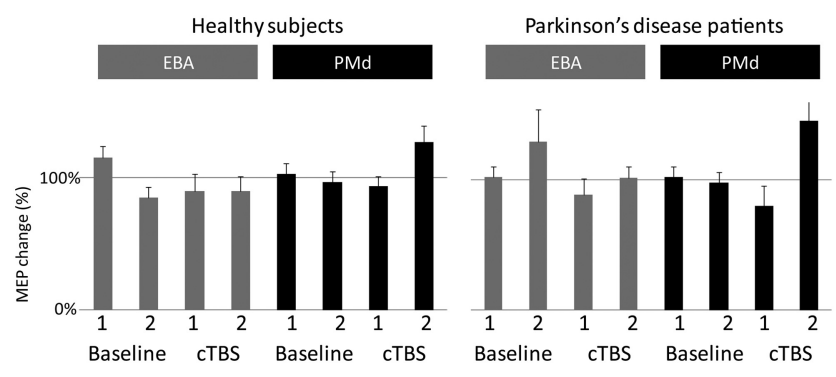

Figure 4. MEPs. Relative change in mean peak-to-peak amplitude of the MEPs, normalized to MEP amplitude measured at baseline. The first post-cTBS measurement (cTBS1) was performed $7 \mathrm{~min}$ after the end of cTBS, and before the onset of the motor imagery task. The second post-cTBS measurement (CTBS2) was performed after the end of the motor imagery task.

supported by our finding that overall imagery performance was comparable between patients and healthy subjects.

\section{A compensatory role for the extrastriate body area in PD}

Inhibiting the right EBA prevented PD patients, but not healthy subjects, from integrating current estimates of the body state into a motor plan. This effect was not a consequence of EBA-driven changes in corticospinal excitability in either group. Yet, the present findings clearly indicate that the EBA can play a role in motor control, adding causal evidence to previous suggestions (Astafiev et al., 2004; Kühn et al., 2011). More precisely, we show that PD patients use the EBA to estimate the current state of the body in space, a necessary requirement for specifying a motor plan suitable to achieve a desired end state (Shadmehr and Krakauer, 2008). It remains unclear how the EBA of PD patients can support this function. One possibility is that the EBA estimates the difference between desired and current body posture, integrating visual and somatosensory information (Zimmermann et al., 2012). In our study, subjects had no visual information about the current orientation of their hands, so PD patients would use the EBA for processing somatosensory information about body parts. However, it is unclear whether the EBA receives somatosensory information. Another possibility is that the EBA effects shown in this study reflect remote alterations conveyed from the EBA into parietal and premotor regions known to be involved in estimating the spatial configuration of the body (de Lange et al., 2006; Helmich et al., 2007). In this scenario, compensatory effects of EBA would arise from enhanced connectivity, rather than enhanced local activity.

\section{Dorsal premotor functionality in PD}

Inhibiting the left PMd prevented healthy subjects, but not PD patients, from integrating current estimates of the body state into a motor plan. We draw three inferences from these observations. First, the left PMd of PD patients was physiologically sensitive to cTBS (as indicated by an MEP reduction), but that intervention did not influence motor imagery performance. This finding suggests that the known hyperactivity of premotor areas in PD (Sabatini et al., 2000; Wu and Hallett, 2005) is more likely to be dysfunctional than compensatory in nature. Second, unilateral inhibition of the left PMd in healthy subjects is sufficient to alter their ability to incorporate the current state of their body into a motor plan, a strong confirmation of the known hemispheric dominance of this frontal region for supporting motor imagery (Haaland et al., 2004; de Lange et al., 2006, 2008). Third, healthy subjects could not recruit compensatory circuits to supplement PMd alterations, as observed in PD patients. This observation suggests that the EBA-based compensatory mechanism found in PD might require time to develop, or that this mechanism becomes effective only once the PMd is functionally disconnected from the posterior parietal regions supporting the incorporation of the current body posture into a motor plan (de Lange et al., 2006).

\section{Interpretational issues}

Matched performance between patient and control groups is an important precondition for isolating compensatory mechanisms (Price and Friston, 2002). In this study, both performance and cTBS effects on cortical excitability were matched across groups at baseline. There was a significant difference in age between groups, and it is possible that control subjects were more sensitive to cTBS stimulation over PMd than PD patients. Elderly subjects might have higher cerebral activation to compensate for agerelated decline in functionality (Ramsøy et al., 2011). However, it is unclear how the age difference alone could account for the double dissociation between behavioral consequences of cTBS on EBA and PMd across groups.

It might be argued that motor imagery is a loosely defined phenomenon that could be solved using a variety of strategies and cerebral mechanisms. In fact, the characteristics of the imagery task used in this study allow for specific inferences. Reaction times increased with increasing stimulus rotation for both hands, indicating that the participants used mental rotation to solve the task, in line with previous findings (Parsons, 1987, 1994; Dominey et al., 1995; de Lange et al., 2006; Helmich et al., 2007). Reaction times were also sensitive to orientation of the stimulus with respect to the body axis, indicating that the participants imagined a movement with the same biomechanical constraints as their own hand. Moreover, reaction times were sensitive to the congruency between the orientation of the hand shown on the screen and the current posture of the subject's (unseen) hand, 
indicating that the computations occurring during motor imagery incorporated the current state of the body.

This study builds on recent findings (Helmich et al., 2007), yet the two studies report different effects. However, these studies differ in a number of procedures, and those inconsistencies are likely related to different sensitivities of the outcome measures to different factors influencing performance of the imagery task. Helmich et al. (2007) focused on the effects of biomechanical constraints on metabolic indexes of cortical activity and connectivity. In contrast, the present study was designed for isolating behavioral effects related to the current body posture. Subjects changed their hand posture every 12 trials. This procedure reduced the chances of habituation of the posture congruency effect, and generated differential reaction time effects between posture-matched and unmatched conditions that were unaffected by time-related effects across experimental sessions. By the same token, this measure does not allow us to specify whether the present findings result from increased reaction times in the posture-matched condition or from decreased reaction times in the posture-unmatched condition. Future studies will need to disambiguate the sources of this effect, possibly by using tasks that dissociate between posture congruency effects on initial and final states of an action (Zimmermann et al., 2012). Another outstanding issue concerns the interference procedure, and in particular the largely unknown physiological effects of cTBS on diseased brains. Continuous TBS produces inhibitory effects when applied over different cortical areas (Franca et al., 2006; Huang et al., 2009; Volman et al., 2011). In this study, MEPs decreased immediately after cTBS over PMd in both the PD group (statistically) and in healthy subjects (numerically) when the baseline data were pooled. Similarly, a recent study demonstrated that cTBS given to the right PMd of healthy subjects did not change the size of MEPs recorded from either left or right abductor pollicis brevis muscle (Stefan et al., 2008). In this study, MEPs increased in both healthy subjects (statistically) and PD patients (numerically) after cTBS was followed by $\sim 25 \mathrm{~min}$ of motor imagery. This effect fits with the well known state dependency of cTBS effects. For instance, 1 min of voluntary contraction of a muscle, during or after cTBS, changes the size and direction of the TBS aftereffects (Huang et al., 2008). This study suggests that motor imagery evokes similar cTBS aftereffects as voluntary contractions, in line with the neurophysiological overlap between motor imagery and movement execution (Jeannerod and Frak, 1999; Cisek and Kalaska, 2004).

\section{Conclusion}

We have shown that patients with PD use a visual cortical area, the extrastriate body area, to influence the motor imagery network that encodes the current state of the body in space during the generation of a motor plan. This compensatory effect might be related to altered PMd functionality in PD, and it might be implemented through changes in long-range connectivity between EBA and PMd (Helmich et al., 2007). These findings provide causal evidence for the compensatory role of a visual cortical region during motor-related processes in $\mathrm{PD}$, opening the way for understanding how the extrastriate body area can improve motor function in this disorder, and whether this improvement is directly related to dopaminergic dysfunction.

\section{References}

Astafiev SV, Stanley CM, Shulman GL, Corbetta M (2004) Extrastriate body area in human occipital cortex responds to the performance of motor actions. Nat Neurosci 7:542-548.
Azulay JP, Mesure S, Blin O (2006) Influence of visual cues on gait in Parkinson's disease: contribution to attention or sensory dependence? J Neurol Sci 248:192-195.

Berardelli A, Rothwell JC, Thompson PD, Hallett M (2001) Pathophysiology of bradykinesia in Parkinson's disease. Brain 124:2131-2146.

Bezard E, Gross CE, Brotchie JM (2003) Presymptomatic compensation in Parkinson's disease is not dopamine-mediated. Trends Neurosci 26:215-221.

Blandini F, Nappi G, Tassorelli C, Martignoni E (2000) Functional changes of the basal ganglia circuitry in Parkinson's disease. Prog Neurobiol 62:63-88.

Braak H, Del Tredici K, Rüb U, de Vos RA, Jansen Steur EN, Braak E (2003) Staging of brain pathology related to sporadic Parkinson's disease. Neurobiol Aging 24:197-211.

Buhmann C, Binkofski F, Klein C, Büchel C, van Eimeren T, Erdmann C, Hedrich K, Kasten M, Hagenah J, Deuschl G, Pramstaller PP, Siebner HR (2005) Motor reorganization in asymptomatic carriers of a single mutant Parkin allele: a human model for presymptomatic parkinsonism. Brain 128:2281-2290.

Cisek P, Kalaska JF (2004) Neural correlates of mental rehearsal in dorsal premotor cortex. Nature 431:993-996.

de Lange FP, Helmich RC, Toni I (2006) Posture influences motor imagery: an fMRI study. Neuroimage 33:609-617.

de Lange FP, Jensen O, Bauer M, Toni I (2008) Interactions between posterior gamma and frontal alpha/beta oscillations during imagined actions. Front Hum Neurosci 2:7.

Dominey P, Decety J, Broussolle E, Chazot G, Jeannerod M (1995) Motor imagery of a lateralized sequential task is asymmetrically slowed in hemiParkinson's patients. Neuropsychologia 33:727-741.

Edelman GM, Gally JA (2001) Degeneracy and complexity in biological systems. Proc Natl Acad Sci U S A 98:13763-13768.

Franca M, Koch G, Mochizuki H, Huang YZ, Rothwell JC (2006) Effects of theta burst stimulation protocols on phosphene threshold. Clin Neurophysiol 117:1808-1813.

Gentner R, Wankerl K, Reinsberger C, Zeller D, Classen J (2008) Depression of human corticospinal excitability induced by magnetic theta-burst stimulation: evidence of rapid polarity-reversing metaplasticity. Cereb Cortex 18:2046-2053.

Haaland KY, Elsinger CL, Mayer AR, Durgerian S, Rao SM (2004) Motor sequence complexity and performing hand produce differential patterns of hemispheric lateralization. J Cogn Neurosci 16:621-636.

Helmich RC, de Lange FP, Bloem BR, Toni I (2007) Cerebral compensation during motor imagery in Parkinson's disease. Neuropsychologia 45:2201-2215.

Huang YZ, Edwards MJ, Rounis E, Bhatia KP, Rothwell JC (2005) Theta burst stimulation of the human motor cortex. Neuron 45:201-206.

Huang YZ, Rothwell JC, Edwards MJ, Chen RS (2008) Effect of physiological activity on an NMDA-dependent form of cortical plasticity in human. Cereb Cortex 18:563-570.

Huang YZ, Rothwell JC, Lu CS, Wang J, Weng YH, Lai SC, Chuang WL, Hung J, Chen RS (2009) The effect of continuous theta burst stimulation over premotor cortex on circuits in primary motor cortex and spinal cord. Clin Neurophysiol 120:796-801.

Jeannerod M, Frak V (1999) Mental imaging of motor activity in humans. Curr Opin Neurobiol 9:735-739.

Keus SH, Munneke M, Nijkrake MJ, Kwakkel G, Bloem BR (2009) Physical therapy in Parkinson's disease: evolution and future challenges. Mov Disord 24:1-14.

Kühn S, Keizer A, Rombouts SA, Hommel B (2011) The functional and neural mechanism of action preparation: roles of EBA and FFA in voluntary action control. J Cogn Neurosci 23:214-220.

Langston JW, Widner H, Goetz CG, Brooks D, Fahn S, Freeman T, Watts R (1992) Core assessment program for intracerebral transplantations (CAPIT). Mov Disord 7:2-13.

Mills KR, Boniface SJ, Schubert M (1992) Magnetic brain stimulation with a double coil: the importance of coil orientation. Electroencephalogr Clin Neurophysiol 85:17-21.

Oldfield RC (1971) The assessment and analysis of handedness: the Edinburgh inventory. Neuropsychologia 9:97-113.

Palop JJ, Chin J, Mucke L (2006) A network dysfunction perspective on neurodegenerative diseases. Nature 443:768-773. 
Parsons LM (1987) Imagined spatial transformations of one's hands and feet. Cogn Psychol 19:178-241.

Parsons LM (1994) Temporal and kinematic properties of motor behavior reflected in mentally simulated action. J Exp Psychol Hum Percept Perform 20:709-730.

Price CJ, Friston KJ (2002) Functional imaging studies of neuropsychological patients: applications and limitations. Neurocase 8:345-354.

Ramsøy TZ, Liptrot MG, Skimminge A, Lund TE, Sidaros K, Christensen MS, Baaré W, Paulson OB, Jernigan TL, Siebner HR (2011) Healthy aging attenuates task-related specialization in the human medial temporal lobe. Neurobiol Aging. Advance online publication. Retrieved November 4, 2011. doi: 10.1016/j.neurobiolaging.2011.09.032.

Sabatini U, Boulanouar K, Fabre N, Martin F, Carel C, Colonnese C, Bozzao L, Berry I, Montastruc JL, Chollet F, Rascol O (2000) Cortical motor reorganization in akinetic patients with Parkinson's disease: a functional MRI study. Brain 123:394-403.

Shadmehr R, Krakauer JW (2008) A computational neuroanatomy for motor control. Exp Brain Res 185:359-381.

Stefan K, Gentner R, Zeller D, Dang S, Classen J (2008) Theta-burst stimulation: remote physiological and local behavioral after-effects. Neuroimage 40:265-274.
Suteerawattananon M, Morris GS, Etnyre BR, Jankovic J, Protas EJ (2004) Effects of visual and auditory cues on gait in individuals with Parkinson's disease. J Neurol Sci 219:63-69.

Urgesi C, Berlucchi G, Aglioti SM (2004) Magnetic stimulation of extrastriate body area impairs visual processing of nonfacial body parts. Curr Biol 14:2130-2134.

Urgesi C, Calvo-Merino B, Haggard P, Aglioti SM (2007) Transcranial magnetic stimulation reveals two cortical pathways for visual body processing. J Neurosci 27:8023-8030.

van Nuenen BF, van Eimeren T, van der Vegt JP, Buhmann C, Klein C, Bloem BR, Siebner HR (2009) Mapping preclinical compensation in Parkinson's disease: an imaging genomics approach. Mov Disord 24 [Suppl 2]:S703-S710.

Volman I, Roelofs K, Koch S, Verhagen L, Toni I (2011) Anterior prefrontal cortex inhibition impairs control over social emotional actions. Curr Biol 21:1766-1770.

Wu T, Hallett M (2005) A functional MRI study of automatic movements in patients with Parkinson's disease. Brain 128:2250-2259.

Zimmermann M, Meulenbroek RG, de Lange FP (2012) Motor planning is facilitated by adopting an action's goal posture: an fMRI study. Cereb Cortex 22:122-131. 